\title{
Randlage mitten in Europa
}

\section{Herausforderungen der Angebotsfinanzierung in Mazedonien}

\section{ELFI HIRSCH}

Elfi Hirsch, Diplom-Sozialpädagogin (BA), studiert derzeit Sozialmanagement. Davor war sie Praktikantin und freiberuflicher Consultant für die Gesellschaft für Internationale Zusammenarbeit für Armenien und Mazedonien.

Elfi.Hirsch@gmx.net

\author{
Die Finanzierung von Nichtregierungsorganisationen \\ als Leistungserbringer sozialer Angebote ist in \\ Europa unterschiedlich geregelt. In Mazedonien \\ beispielsweise stellt der Staat nur geringe finanzielle \\ Ressourcen für freie Anbieter zur Verfügung, \\ so dass diese Organisationen vor allem auf \\ internationale Geldgeber angewiesen sind.
}

Seit der Wirtschaftskrise in Europa wird in vielen Ländern von Kürzungen gesprochen, die sich zunehmend auf alle Bereiche ausweiten. So sind auch Renten- und Sozialsysteme im gesamten europäischen Raum von der wirtschaftlichen und finanzpolitischen Situation betroffen. Wie sich die Situation entwickeln wird, ist nur zu vermuten. Klar ist jedoch schon heute, dass die Finanzierung sozialer Angebote gesichert werden muss, wozu vermehrt Kreativität und Innovation benötigt werden.

Im Zuge steigender Preise stehen Kommunen vor schmaler werdenden Kassen. Die Folge daraus sind Kürzungen staatlicher Zuwendungen. Institutionelle Förderung wird durch Projektförderung ersetzt. Vor allem dort, wo Angebote nicht genau einer Person zugeordnet werden können, kein Rechtsanspruch besteht oder die Nachsorge und Prävention nicht finanziell abgesichert sind, ist eine Projektfinanzierung hinderlich für die Entwicklung einer Organisationsstruktur und bedarfsgerechter Angebote.

Schauen wir über die Grenzen Deutschlands, ist erkennbar, dass unsere wachsenden Probleme woanders schon den Alltag ausmachen. Die Erschließung alternativer Finanzierungsformen, die über staatliche Zuwendungen, Spenden, Sponsoring, Stiftungen oder Eigenbeiträge hinausgehen, wird zunehmend notwendig.

In Deutschland werden Angebote bislang vorrangig öffentlich über Verträge zwischen Leistungsanbietern und staatlichen Institutionen finanziert. Kennzeichnend für die heutige Finanzierung sozialer Einrichtungen in Deutschland ist der Finanzierungsmix aus hauptsächlich öffentlichen, selbst erwirtschafteten und philanthropischen Mitteln. Mit diesem Mix einher gehen:

- Maßnahmen zur Kostenreduzierung, - die Notwendigkeit messbarer Ergebnisse,

- Case Management,

- One-Stop-Zentren und

- die Einbeziehung unterschiedlicher Akteure.

In Deutschland sowie in anderen europäischen Ländern sehen Einrichtungen sich zunehmend in der Notwendigkeit, zur Kostendeckung neben den bisher gängigen Finanzierungsquellen Alternativen zu identifizieren. Diese sogenannte Drittmittelakquise soll zu einem dauerhaften Bestehen sozialer Einrichtung beitragen, jedoch nicht die staatliche Fürsorgepflicht ersetzen. Da nicht-staatliche Einrichtungen auch Leistungen im Sinne der Sozialpolitik erbringen, ist eine staatliche Grundfinanzierung eine Voraussetzung zur Erfüllung dieser Aufgaben. Die Drittmittelakquise soll vor allem die Flexibilität und Qualität der Einrichtung erhöhen.

Trotz steigender Ausgaben im sozialen Bereich, werden in Deutschland öffentliche Zuwendungen gestrichen und soziale Einrichtungen müssen ihr Repertoire kürzen. Obwohl Rechtsan- 
sprüche bestehen, können somit Angebote nicht mehr flächendeckend oder im ausreichenden Maße angeboten werden - ein Phänomen, das beispielsweise in Mazedonien Alltag ist.

\section{Beispiel Mazedonien}

Angebote des öffentlichen Sektors richten sich vor allem an die Deckung besonderer Bedarfe, die durch die staatliche Fürsorge nicht erfasst werden. Diese oft interdisziplinären Angebote richten sich an identifizierte Bedarfe und bieten Problemlösungsansätze für gesellschaftliche Probleme, die sich im Zuge des gesellschaftlichen Wandels ständig verändern. Stabile Finanzierungen gewährleisten eine langfristige, strategische Planung der sozialen Einrichtung und ihres Angebots sowie Flexibilität in der Anpassung an sich wandelnde Bedarfe in der Gesellschaft. Die Organisation wird in die Lage versetzt, sich bedarfsgerecht weiterzuentwickeln und ständig zu verbessern.

Bisher richteten sich in Mazedonien die Prioritäten und Angebot an Fördermöglichkeiten und Projektausschreibungen internationaler Geberorganisationen, die eine kurz- oder mittelfristige Finanzierung der Einrichtung gewährleisteten. Eine Spezialisierung auf eine bestimmte Zielgruppe und eine Ausrichtung auf ein bestimmtes Angebot, das auf identifizierte Bedarfe der Gesellschaft reagiert, ist oft aus finanziellen Gründen eingeschränkt oder nicht möglich. Langfristige Planungen, Leitbildgestaltungen, Organisationsentwicklungen sowie das dauerhafte Bestehen sozialer Einrichtungen sind durch unsichere Finanzierungsgrundlagen gefährdet.

\section{Mazedonien - ein kurzes Porträt}

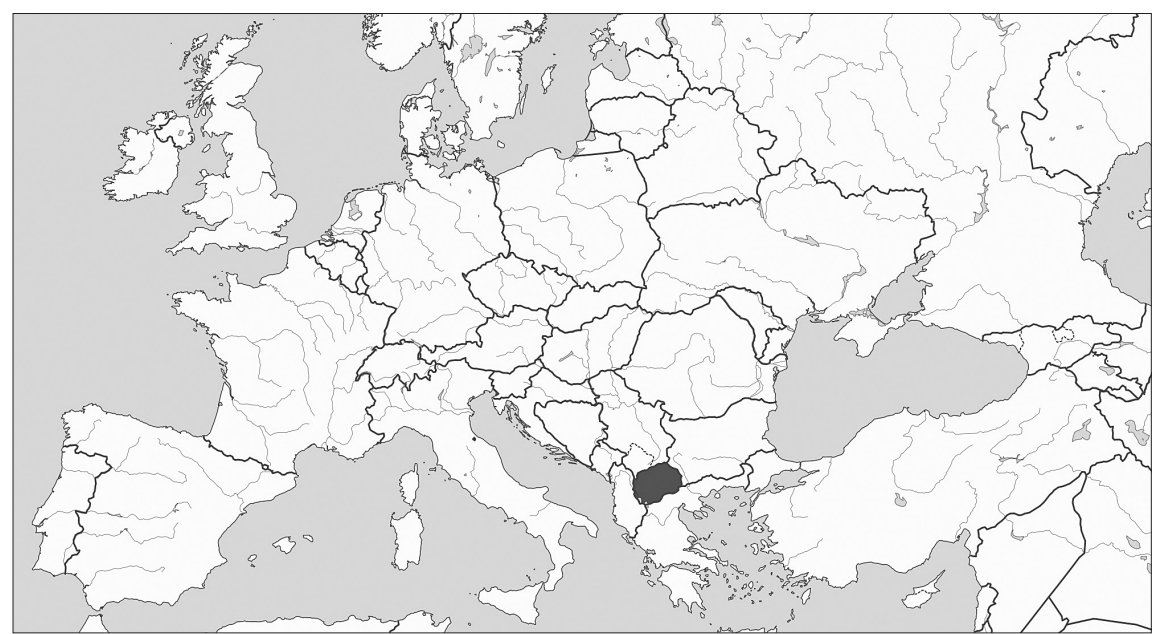

Mazedonien, auch Makedonien, amtlich Republik Mazedonien oder Republik Makedonien ist ein Binnenstaat in Südosteuropa. Aufgrund des Namensstreits mit dem südlichen Nachbarn Griechenland wird er auch als "Ehemalige Jugoslawische Republik Mazedonien « bezeichnet. Mazedonien bestand $a b 1946$ als südlichste Teilrepublik des sozialistischen Jugoslawien und rief 1991 seine Unabhängigkeit aus. Seit Dezember 2005 hat Mazedonien den Status eines Beitrittskandidaten der Europäischen Union. Mazedonien hat eine der schwächsten Volkswirtschaften Europas und befindet sich in einem Transformierungsprozess, sowohl wirtschaftlich als auch politisch. Das Land hat mit hohen Arbeitslosenzahlen und einer schwachen Infrastruktur sowie fehlenden Investitionen zu kämpfen. Mazedonien hat etwas mehr als zwei Millionen Einwohner. Die Bevöl- kerung ist ethnisch gemischt. Neben der größten Volksgruppe, den Mazedoniern, die etwa 64 Prozent der Gesamtbevölkerung stellen, gibt es eine große Minderheit an Albanern (25\%). Auch kleine Minderheiten von Türken (3,85\%), Roma (2,66 $\%)$, Serben (1,78\%), Bosniaken (0,84\%) und Aromunen/Meglenorumänen $(0,48$ $\%)$ und anderen $(1,04 \%)$ sind vorhanden. Durch diese Situation gab und gibt es immer wieder ethnisch motivierte Konflikte, vor allem zwischen Mazedoniern und Albanern. Nach den bürgerkriegsähnlichen Zuständen 2001 und dem danach unterschriebenen Friedensvertrag hat sich die Gesamtlage der Volksminderheiten im Land deutlich verbessert. Mit einer gesellschaftlichen Gleichstellung ist jedoch immer noch nicht zu rechnen.

Quelle: www.wikipedia.org
Vor allem Einrichtungen, deren Betreuungsangebot stark abhängig sind von sozioökonomischen Entwicklungen und den individuellen Bedarfen der Klienten, leiden unter der Finanzierungsproblematik und dem Druck, die Angebote möglichst gering zu halten. Qualität, die von der Qualifikation des Personals abhängt und von der Möglichkeit, flexibel auf Veränderungen zu reagieren, kann somit nicht gewährleistet werden.

\section{Partnerarbeit und Netzwerke als Finanzierungsquelle}

Einem Bericht der internationalen Arbeitsorganisation (ILO) über die allgemeine Erbringung sozialer Angebote auf dem Westbalkan von 2004 zufolge bestehen Mängel im mazedonischen Sozialsystem:

- Eine allgemeine Strategie zur Entwicklung von Partnerschaftsmodellen zwischen dem nichtstaatlichen Sektor und dem Staat fehlt.

- Rechtliche und regulatorische Rahmenbedingungen in Bezug auf die Ernennung von Nichtregierungsorganisationen zu Dienstleistungsanbietern, Bedingungen zur Leistungserbringung z. B. Akkreditierungsverfahren, Überwachung, Messung und Bewertung der Maßnahmen) sowie Kriterien und Verfahren zu Finanzierungsformen, einschließlich dem Zuwendungsverfahren und den Durchführungsvereinbarungen sind unzureichend.

Ein neuerer Bericht der US-amerikanischen Regierung aus dem Jahr 2012 hebt hervor, dass es zwar formal Kooperationen zwischen staatlichen und nichtstaatlichen Institutionen zur Leistungserbringung gibt, der Staat jedoch bisher sehr geringe finanzielle Ressourcen zur Verfügung stellt sowie wenige Anstrengungen im Bereich der Wirkungsmessung und Berichterstattung unternimmt.

Es wird deutlich, dass der mazedonische Staat die Mindestanforderungen in der Erbringung sozialer Dienste aufgrund des internationalen Drucks erfüllen möchte, jedoch wenige Mittel dafür zur Verfügung stellt. Daraus ergibt sich das Hauptproblem in der nicht vorhandenen langfristigen und nachhaltigen Finanzierung. Potentiale bestehen in der Anknüpfung an vorhandene Strukturen (vgl. Abb. Seite 181). 


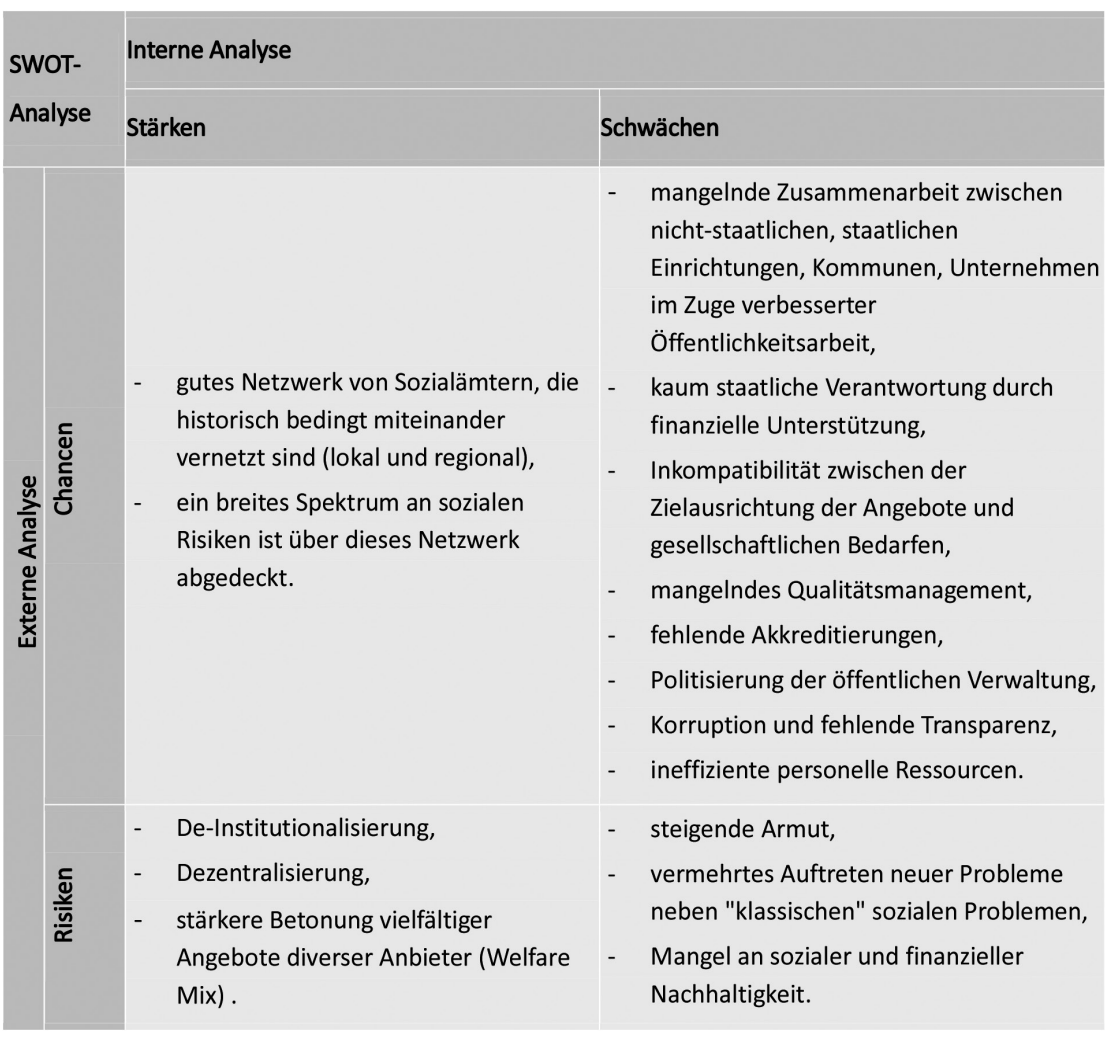

Die vorhandenen Stärken sozialer Organisationen in Mazedonien leiden vor allem an der fehlenden langfristigen Finanzierung der Angebote.

Einrichtungen in Mazedonien können sich als Nichtregierungsorganisation registrieren lassen und unterliegen dann dem öffentlichen Haushaltsrecht. Für eine nachhaltige und effektive Partnerschaft zwischen staatlichen und nichtstaatlichen Institutionen sind Akkreditierungsmechanismen notwendig, die einerseits die Zusammenarbeit zwischen Kommunen, Sozialämtern und der Einrichtungen zur Bereitstellung sozialer Angebote definieren, andererseits Kriterien, Standards und Berichterstattung festlegen.

Eine finanzielle Unterstützung der Einrichtungen sollte durch den Staat gesichert werden. Ferner sollte ein klarer rechtlicher Rahmen bezogen auf die Organisationsentwicklung, Leistungen und Kooperationsmöglichkeiten mit der Wirtschaft für nichtstaatliche Einrichtungen geschaffen werden.

\section{Internationale Geber und mangelnde Nachhaltigkeit}

Anders als in Deutschland, wo viele Organisationen über öffentliche Zuwendungen (teil-) finanziert werden, besteht in Mazedonien die Problematik, dass trotz bestehenden Rechtsanspruchs der
Staat die Finanzierung nichtstaatlicher sozialer Angebote kaum bis gar nicht wahrnimmt.

Feste Partnerstrukturen und gezielte Öffentlichkeitsarbeit sind bedeutend für die Netzwerkarbeit und Mittelakquise. Die wichtigsten Partner der Nichtregierungsorganisationen sind Kommunen, ausländische Organisationen und Stif-
Auftraggebern selbst durchgeführt. Projektfinanzierungen durch internationale Geberorganisationen übernahmen bisher 90 bis 100 Prozent der Kosten. Nur 25 bis 40 Prozent der Einnahmen stammten aus Eigenbeiträgen, Spenden und staatlichen Zuwendungen.

Die Nachhaltigkeit dieser Finanzierungsform ist gering; Langzeitfinanzierungen und langfristige Planungen unmöglich, bis auf die wenigen Ausnahmen, die dauerhafte Vereinbarungen mit internationalen Geberorganisationen geschlossen haben. Übergeordnetes Ziel internationaler Geberorganisationen ist die kurzfristige Unterstützung, um den Staat die Notwendigkeit des Angebots vor Augen zu führen, so dass dieser die Verantwortung für Angebote der sozialen Sicherung im eigenen Land übernimmt. Hierin begründet sich die kurz- und mittelfristige Projektfinanzierung internationaler Geber.

Im internationalen Dialog ist die Finanzierung über internationale Geber umstritten. Sie stellt keine nachhaltige Alternative zu staatlichen Zuwendungen dar und kann die Bemühungen des eigenen Staates behindern. So ist auch in Mazedonien zu beobachten, dass der Staat ein geringes Interesse im Bereich der Förderung nichtstaatlicher Einrichtungen zeigt, weil er annimmt, dass diese finanziell durch internationale Organisationen unterstützt werden. Ferner muss sich an Vorgaben der Geberorganisation bei der Implementierung, beim Monitoring sowie der Evaluation der Projekte gehalten werden. Die un-

\section{"Internationale Geberorganisationen konzentrieren sich in Mazedonien auf kurzfristige Unterstützungen«}

tungen. Unternehmen und Ministerien gehören zur Partnerstruktur, tragen jedoch wenig zur Liquidität bei.

Bevor sich internationale Geberorganisationen immer mehr aus Mazedonien zurückzogen, waren diese die Hauptfinanzierungsquelle sozialer Angebote. Der geringe Anteil staatlicher Förderung war bisher unbedeutend für die Projektdurchführung. Die Projektanträge wurden in der Regel direkt beim Geber gestellt. Ergebnis- und Wirkungsmonitoring wurden von den abhängige Organisationsentwicklung sowie die Zusammenarbeit mit lokalen Organisationen und Kommunen wird behindert, vor allem durch die Ausrichtung auf die englische Sprache.

\section{Innovation und Kreativität als Lösungsansätze}

Bisher sind Maßnahmen im Bereich der Zusammenarbeit mit der Privatwirtschaft, Unternehmen und Privatpersonen beispielsweise über Corporate 
Social Responsisbility in Mazedonien selten und in Deutschland nur von sehr großen Organisationen genutzt. Während in Deutschland die Quersubventionierung über Zweckbetriebe eine gängige Methode zur Eigenmittelbeschaffung darstellt, ist diese Möglichkeit in Mazedonien noch nicht ausgeschöpft.

Gute Praxisbeispiele aus der Region sind in Bosnien und Herzegowina zu finden. Über Netzwerke in der Region können Praxisbeispiele und Erfahrungen ausgetauscht werden. Doch bedarf die Identifikation und Nutzung alternativer Finanzierungsformen einerseits Kreativität und Innovation sowie feste rechtliche Grundlagen voraus, andererseits Engagement seitens der Einrichtung - in Mazedonien und in Deutschland.

\section{Literatur}

Cekov, Aleksandar \& Taseski, Filip (2009). Combating the Trafficking in Human Beings. Policies of the Republic of Macedonia Reviewed. Analytica thinking laboratory. www.analyticamk. org/files/ReportNo28.ppdf (13.04.2012).

Donevska, Maria \& Gjorgjev, Dragan \& Gerovska Mitev, Maja \& Kalovska, Tanja (2007). Social Protection and Social Inclusion in the former Yugoslav Republic of Macedonia. Summary. European Commission - Directorate General for Employment, Social Affairs and Equal Opportunities Unit E2. Euro Balkan Institute. Fultz, Elaine \& Tracy, Martin (2004). Good Practices in Social Service Delivery in South East Europe. Internationale Labour Organisation. Budapest, International Labour Office. FUNDSFORNGOs, LLC. www.fundsforngos.org/ alternative-fundraising /alternative-fundraisingfor-ngos-1-current-funding-challenges-forngos/\#ixzz213yEShgQ (19.07. 2012).

Hirsch, Elfi (2012). Finanzierungsmechanismen in der Sozialwirtschaft am Beispiel einer europäischen Resozialisationseinrichtung. Hausarbeit Paritätische Bundesakademie. Konrad-Adenauer-Stiftung. Soziale Marktwirtschaft - Garant für wirtschaftlichen Erfolg und soziale Stabilität. www.kas.de/wf/de/71.10269/ (20.07.2012).

La Strada. www.lastrada.org.mk/content_eng. asp?id=21\#Scene_1 (18.6.2011).

Ulrich, Mareen (2008). Fundraising in der sozialen Arbeit. Konzepterstellung für den Unabhängigen Frauenverein e. V. Rathenow. Bachelorarbeit Alice Salomon Hochschule. United States of America (2012). Trafficking in Persons Report. June 2012. Department of State.

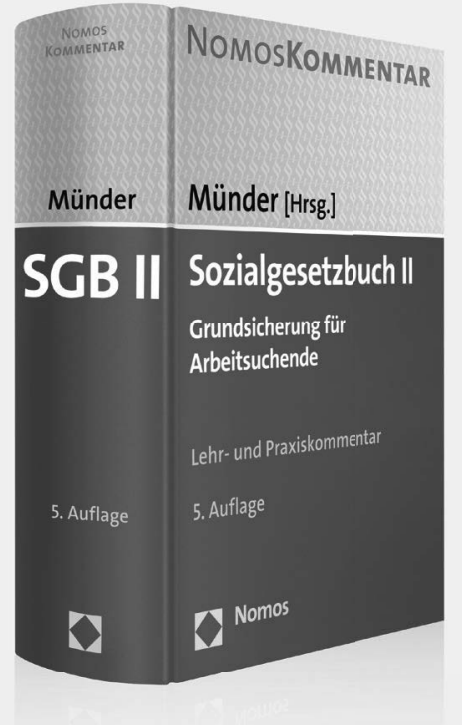

Neuauflage

\section{Sozialgesetzbuch II}

Grundsicherung für Arbeitsuchende

Lehr- und Praxiskommentar

Herausgegeben von

Prof. Dr. Johannes Münder

5. Auflage 2013, 1.212 S., geb., 58,- $€$

ISBN 978-3-8487-0596-2

Der „Münder“ ist das Standardwerk zum Recht der Grundsicherung. Er schafft Klarheit für die praktische Rechtsanwendung, und das zu einem erschwinglichen Preis.

Der Gesetzgeber hat seit der Vorauflage erneut wesentliche Änderungen am SGB II vorgenommen, u.a. durch:

- das Gesetz zur Verbesserung der Eingliederungschancen am Arbeitsmarkt („Instrumentengesetz“)

- das Haushaltsbegleitgesetz 2013

- das Gesetz zur Änderung des Zweiten Buches Sozialgesetzbuch und anderer Gesetze vom 7. Mai 2013.

Mit den Neuerungen, insbesondere dem „Instrumentengesetz“, wurde eine Vielzahl der Leistungen zur Eingliederung in Arbeit deutlich geändert, z.T. gestrichen. So entfällt der Zuschuss für freiwillige Beiträge zur gesetzlichen Rentenversicherung, die Bundeszuschüsse an den Gesundheitsfonds der gesetzlichen Krankenversicherung und an die allgemeine Rentenversicherung werden gekürzt. Bestimmungen des Bildungsteilhabepakets wurden an die Praxisanforderungen angepasst.

"...richtig gelungener Kommentar. Seine Aktualität, der strukturierte Aufbau und die Auslegungs- und Argumentationshilfen in klarer Sprache machen ihn zu einem zuverlässigen Nachschlagewerk."

Jens Jenau, Sozialrecht + Praxis 7/12, zur Vorauflage

Bestellen Sie jetzt telefonisch unter 07221/2104-37.

Portofreie Buch-Bestellungen unter www.nomos-shop.de/21213

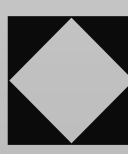

Nomos 